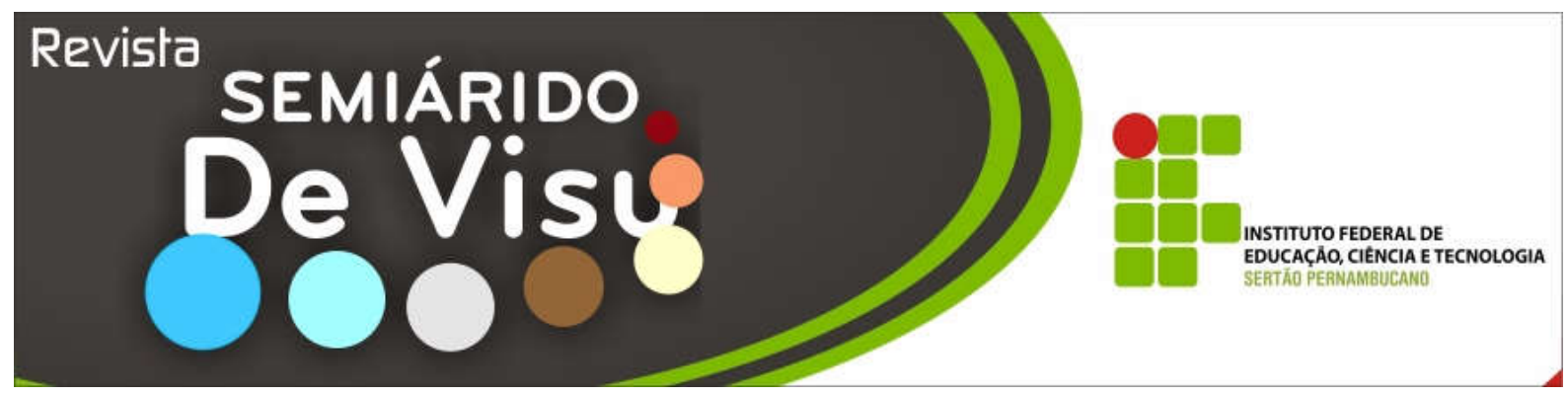

\title{
Obtenção de um modelo dinâmico do crescimento da bactéria Aeromonas hydrophila a partir de dados isotérmicos
}

\author{
Marcos dos Santos Lima ${ }^{1 *}$, Páulia Maria Cardoso Lima Reis ${ }^{1}$, Gláucia Maria Falcão Aragão ${ }^{2}$ \\ 1Instituto Federal do Sertão Pernambucano, Campus Petro lina, Coordenação de Tecnologia de Alimentos, Jardim São Paulo - 56314- \\ 520, Petrolina, PE, Brasil \\ ${ }^{2}$ Uni versidade Federal de Santa Catarina, Departamento de Engenharia de Alimentos, Campus Universitário, Trindade - 88040-900, \\ Florianópolis, SC, Brasil \\ *Autor para corre spondência. Fone: (87) 2101-4300 Ramal 4330 Email: marcos.santos@ifsertao-pe.edu.br
}

\begin{abstract}
RESUMO: Nos últimos anos um esforço considerável foi investido no desenvolvimento de modelos matemáticos que expliquem a dinâmica microbiana em produtos alimentícios. Para que esses modelos possam ser aplicados em alimentos armazenados em condições reais, onde a temperatura varia com o tempo, é necessário considerar o efeito de mudanças em variáveis como temperatura. O objetivo deste trabalho foi o de avaliar a metodologia proposta por Corradini e Peleg (2005) e Corrad ini et al. (2006) para obtenção de um modelo dinâmico a partir de dados isotérmic os do crescimento da bactéria Aeromonas hydrophila, ajustados pelo modelo de Gompertz modificado. Os dados do crescimento isotérmicos foram obtidos no banco de dados ComBase Predictor e para construção dos modelos secundários foram utilizadas as funções matemáticas "Power" e "Power 1" com auxílio do programa Matlab®. O modelo de Gompertz modificado se ajustou bem aos dados is otérmicos de crescimento e a utilização das equações "Power" e "Power 1" no ajuste do modelo secundário apresentou bons resultados, sendo possível a obtenção de um modelo dinâmico a partir da metodo logia proposta por Corradini e Peleg (2005) e Corradini et al. (2006).
\end{abstract}

Palavras-chave: Mic robiologia preditiva; modelagem matemática; Modelo não isotérmico.

\section{Estimation of non-isothermal growth of bacteria Aeromonas hydrophila from isothermal data}

\begin{abstract}
In recent years considerable effort has been invested in the development of mathematical models to explain the growth of microorganisms in food products. For these models may be applied in foods stored in real conditions is necessary to consider the effect of changes in variables such as temperature. The objective of this study was to evaluate the methodology proposed by Corrad ini and Peleg (2005) and Corradini et al. (2006) to obtain a nonisothermal model from isothermal growth data of the bacterium Aeromonas hydrophila, adjusted by Gompertz modified model. The is othermal growth data were obtained from the database ComBase Predictor and secondary models were obtained with the mathematical functions "Power" and "Power 1". The modified Gompertz model fits well to the data of isothermal growth of the bacterium Aeromonas and use of functions "Power" and "Power 1" in the secondary model adjustment showed good results. Was possible to predict the non-is othermal growth of Aeromonas hydrophila bacteria using the methodology proposed by Corradini and Peleg (2005) and Corradini et al. (2006).
\end{abstract}

key words: Predic tive mic robiology, Mathematical modeling, Non-iso thermal model. 


\section{Introdução}

$\mathrm{O}$ comportamento dos microrganismos nos alimentos (crescimento, sobrevivência e morte) é determinado pelas propriedades que este possui e por condições de estocagem. A microbiologia preditiva está baseada na hipótese de que o efeito dessas propriedades pode ser previsto por meio de modelos matemáticos derivados de estudos quantitativos dos microrganismos (Nakashima et al., 2000).

Nos últimos anos um esforço considerável foi investido no desenvolvimento de modelos matemáticos que expliquem a dinâmica microbiana em produtos alimentícios, e uma série de modelos e expressões matemáticas para predição do crescimento microbiano em alimentos foram desenvolvidos (Baranyi \& Roberts, 1995; Buchanan et al., 1997; Van Impe et al., 2005). Hoje, a microbiologia preditiva tem sido amplamente utilizada para modelar o crescimento de bactérias em função de fatores ambientais como temperatura, $\mathrm{pH}$ e Aw, também podendo ser empregada para prever a vida de prateleira dos produtos (Cayré et al., 2005; Slongo et al., 2009).

Em condições isotérmicas (temperaturas constantes) as curvas de crescimento bacteriano são, geralmente, em forma de sigmóide e podem ser descritas por vários modelos matemáticos. Um dos principais modelos de crescimento é o de Gompertz modificado (Gibson et al.,1987), expresso na equação 01 (Eq. 01).

$\log \left(\frac{N}{N_{0}}\right)=A \times \exp \left\{-\exp \left[\frac{\mu \times e}{A}(\lambda-t)+1\right]\right\}$

(Eq. 01)

Sendo,

$\lambda$ - duração da fase lag;

$\mu$ - velocidade específica de crescimento; e

A - início da fase estacionária. microbiano

Modelos secundários de crescimento
Variações da população de microrganismos nos alimentos em função do tempo (cinética microbiológica) são influenciadas pelas condições de estocagem (fatores extrínsecos) e pelas características do produto (fatores intrínsecos). Estes fatores, coletivamente, são denominados parâmetros ambientais. Modelos matemáticos que descrevem o efeito das condições ambientais sobre os valores de parâmetros dos modelos primários são denominados modelos secundários. Os modelos secundários envolvem equações que descrevem a variação das respostas dos modelos primários (duração da fase lag, velocidade específica de crescimento e densidade máxima de população) em função de modificaçõ es nos fatores ambientais (Sarmento, 2006).

microbiano

Modelos dinâmicos do crescimento

Um dos componentes mais importantes na avaliação quantitativa do risco microbiológico em alimentos é o desenvolvimento de modelos matemáticos de predição do cres cimento microbiano capazes de levar em consideração a influência da variação de parâmetros ambientais sobre o crescimento do microrganismo ao longo da cadeia de abastecimento (Nauta, 2002; Nauta et al., 2005). Existem diversos modelos matemáticos que permitem predizer o crescimento de uma ampla classe de microrganismos patogênicos ou deteriorantes sobre combinações distintas de fatores ambientais intrínsecos ou extrínsecos. Onde a modelagem matemática normalmente é realizada assumindo condições constantes em fatores ambientais para se determinar os parâmetros cinéticos de crescimento (Baranyi et al., 1995; Bobelyn et al., 2006; Sarmento, 2006). Portanto, para que os modelos matemáticos possam ser aplicados em alimentos armazenados em condições reais, onde a temperatura varia em função do tempo, é necessário considerar no modelo o efeito das mudanças de variáveis externas sobre o crescimento microbiano para que as predições 
sejam mais precisas a fim de assegurar a vida útil dos mesmos (Cayré et al., 2003).

Um dos principais fatores ambientais que afeta diretamente o crescimento microbiano nos alimentos é a temperatura. A temperatura dos alimentos durante a es tocagem, distribuição, e armazenamento, está constantemente sujeita a alterações. Modelos efetivos que possam descrever o crescimento microbiano sobre condições dinâmicas de temperatura, ou seja, com variação de temperatura em função do tempo, são necessários para aplicações práticas (Fujikawa et al., 2004).

O modelo matemático proposto por Corradini e Peleg (2005) e Corradini et al., (2006) demonstra que modelos primários e secundários, derivados de dados de crescimento em temperatura constante (isotérmicos), podem ser usados para predizer padrões do modelo de crescimento microbiano sob variação de temperatura (não isotérmico). Estes autores propuseram uma nova versão do Modelo Logístico como modelo primário de crescimento e chamaram de Modelo Logístico Modificado (Eq. 02 e 03).

$$
y(t)=\frac{a^{\prime}(T)}{1+\exp \left\{k(T) t_{c}(T)-t\right\}}-\frac{a^{\prime}(T)}{1+\exp \left[k(T) t_{c}(T)\right]}
$$

(Eq. 02)

Sendo y $(t)=\log$ N/No; logo:

$$
\frac{d y(t)}{d t}_{(\text {Tcte })}=\frac{k(T) a(T) \exp \left(k(T)\left\{t_{c}(T)-t\right\}\right)}{\left[1+\exp \left(k(T)\left\{t_{c}(T)-t\right\}\right)\right]^{2}}
$$

(Eq. 03)

Onde os parâmetros do modelo são:

$\mathrm{a}=$ população máxima atingida;

$\mathrm{k}=$ Velocidade específica máxima de crescimento $\left(\mathrm{h}^{-1}\right)$;

tc $=$ Ponto de inflexão na curva $(h)$.

Estes parâmetros (a, k e tc) podem ser descritos em função da temperatura utilizando modelos secundários obtidos de equações exponenciais, polinomiais, linear, potência, conforme o melhor ajuste aos dados propostos, considerando que:

$\mathrm{a}(\mathrm{T})=\mathrm{a}[\mathrm{T}(\mathrm{t})] ; \quad($ Eq. 04)

$\mathrm{k}(\mathrm{T})=\mathrm{k}[\mathrm{T}(\mathrm{t})] ; \quad($ Eq. 05)

tc $(\mathrm{T})=\operatorname{tc}[\mathrm{T}(\mathrm{t})] .($ Eq. 06)

Se incluído os parâmetros descritos nas equações 04, 05 e 06, na equação 03, o modelo não isotérmico pode ser representado pelas equações 07 e 08. Sendo esta a metodologia proposta por Corradini e Peleg (2005) e Corradini et al., (2006) para a obtenção de modelos dinâmicos.

$$
\frac{d y(t)}{d t}=\frac{k[T(t)] a^{\prime}[T(t)] \exp \left(k[T(t)]\left\{t_{c}[T(t)]-t^{*}\right\}\right)}{\left[1+\exp \left(k[T(t)]\left[t_{c}[T(t)]-t^{*}\right)\right]^{2}\right.}
$$

(Eq. 07)

Sendo,

$$
t^{*}=\frac{1}{k(T)} \log e\left[\frac{\exp \left[k(T) t_{c}(T)\right]\left(a^{\prime}(T)+y(T)\left\{1+\exp \left[k(T) t_{c}(T)\right]\right\}\right)}{a^{\prime}(T) \exp \left[k(T) t_{c}(T)\right]-y(t)\left\{1+\exp \left[k(T) t_{c}(T)\right]\right\}}\right]
$$

(Eq. 08)

\section{Aeromonas ssp.}

As bactérias do gênero Aeromonas spp. estão associadas a uma série de doenças e representam um grande perigo à saúde pública, pois se trata de um patógeno emergente relacionado com o aumento da ocorrência de casos de doenças na população. Algumas espécies de Aeromonas móveis podem causar diarréia auto-limitada, infecção de pele e ouvido, e até necrose e septicemia em indivíduos com fatores predisponentes como: cortes, perfurações, cirurgias, distúrbios imunológicos, ou que tiveram prévia exposição a ambientes contaminados, principalmente, água e alimentos consumidos crus (como sashimi) ou mal cozidos (Azevedo et al., 2003).

\section{Objetivo}

O objetivo deste trabalho foi o de avaliar a metodologia proposta por Corradini e Peleg (2005) e Corradini et al. (2006) para 
(LIMA; REIS, ARAGÃO, 2015)

obtenção de um modelo dinâmico a partir de dados isotérmicos do crescimento da bactéria Aeromonas hydrophila, ajustados pelo modelo de Gompertz modificado.

\section{Materiais e Métodos}

Os dados isotérmicos utilizados neste trabalho foram obtidos na base de dados ComBase Predictor. Dados de crescimento da bactéria Aeromonas hydrophila foram coletados das temperaturas de $15,20,25$ e $30^{\circ} \mathrm{C}$ (Tabela 1) fixando-se os fatores de crescimento em: Contagem inicial $\left(\mathrm{UFC} \mathrm{g}^{-1}\right)=0$; fator de crescimento $=0,007 ; \mathrm{pH}=5,8$ e percentual de $\mathrm{NaCl}=2,0$. Para o ajuste dos dados isotérmicos de crescimento foi aplicado o modelo de Gompertz modificado (Gibson et al.,1987), como modelo primário, com auxílio do programa Matlab ${ }^{\circledR}$ (Math Works, Natick, MA, USA). A verificação do ajuste do modelo aos dados de crescimento foi realizada pela utilização do coeficiente de correlação $\left(\mathrm{R}^{2}\right)$ e do erro méd io quadrático (MSE) (Eq. 09).
$M S E=\frac{R S S}{n}=\sum \frac{\left(\text { Valor }_{\text {observado }}-\text { Valor }_{\text {predito }}\right)^{2}}{n}$ (Eq. 09)

Sendo: $\mathbf{R S S}=$ soma dos quadrados residuais $; \mathbf{n}$ $=$ número de graus de liberdade.

Da aplicação do modelo de Gompertz modificado nos dados de crescimento em temperaturas isotérmicas de $15,20,25$ e $30^{\circ} \mathrm{C}$ obtiveram-se os parâmetros $\mathrm{A}, \lambda$ e $\mu$, que correspondem respectivamente, a início da fase estacionária, duração da fase lag e velocidade específica de crescimento, sendo estes parâmetros utilizados para construção dos modelos secundários em função da temperatura, utilizando as equações lineares que melhor se ajustaram aos dados. A partir das equações obtidas nos modelos secundários obteve-se o modelo dinâmico, se aplicando a metodologia proposta por Corradini e Peleg (2005) e Corradini et al. (2006), com o auxílio do programa Matlab ${ }^{\circledR}$.

Tabe la 1. Dados isotérmicos de cresc imento da bac téria Aeromonas hydrophila nas temperaturas de $15,20,25$ e $30^{\circ} \mathrm{C}$. Fonte: (COMBASE, 2011).

\begin{tabular}{|c|c|c|c|c|}
\hline \multirow{3}{*}{ Tempo (h) } & \multicolumn{4}{|c|}{ Crescimento UFC $\mathrm{g}^{-1}(\log \mathrm{N})$} \\
\hline & \multicolumn{4}{|c|}{ Temperatura } \\
\hline & $15^{\circ} \mathrm{C}$ & $20^{\circ} \mathrm{C}$ & $25^{\circ} \mathrm{C}$ & $30^{\circ} \mathrm{C}$ \\
\hline 0 & 0 & 0 & 0 & 0 \\
\hline 4,8 & 0,01 & 0,02 & 0,05 & 0,06 \\
\hline 9,6 & 0,03 & 0,15 & 0,47 & 0,64 \\
\hline 14,4 & 0,1 & 0,63 & 1,52 & 1,87 \\
\hline 19,2 & 0,27 & 1,43 & 2,73 & 3,2 \\
\hline 24 & 0,58 & 2,31 & 3,95 & 4,53 \\
\hline 28,8 & 1,02 & 3,19 & 5,14 & 5,8 \\
\hline 33,6 & 1,51 & 4,08 & 6,23 & 6,79 \\
\hline 38,4 & 2,02 & 4,95 & 6,98 & 7,25 \\
\hline 43,2 & 2,54 & 5,78 & 7,29 & 7,37 \\
\hline 48 & 3,06 & 6,5 & 7,37 & 7,39 \\
\hline 50,4 & 3,32 & 6,78 & 7,38 & 7,39 \\
\hline 55,2 & 3,84 & 7,15 & 7,39 & - \\
\hline 57,6 & 4,1 & 7,25 & - & - \\
\hline 62,4 & 4,61 & 7,35 & - & - \\
\hline 67,2 & 5,11 & 7,38 & - & - \\
\hline 72 & 5,6 & 7,39 & - & - \\
\hline 79,2 & 6,27 & 7,39 & - & - \\
\hline 84 & 6,64 & - & - & - \\
\hline 88,8 & 6,93 & - & - & - \\
\hline 93,6 & 7,13 & - & - & - \\
\hline 98,4 & 7,25 & - & - & - \\
\hline 103,2 & 7,32 & - & - & - \\
\hline 108 & 7,35 & - & - & - \\
\hline 112,8 & 7,37 & - & - & - \\
\hline
\end{tabular}




\begin{tabular}{l|l|l|l|l}
117,6 & 7,38 & - & - & - \\
\hline
\end{tabular}

\section{Resultados e Discussão}

A figura 1 apresenta as curvas de crescimento isotérmico da bactéria Aeromonas hydrophila nas temperaturas de 15, 20, 25 e $30^{\circ} \mathrm{C}$, e demonstram que o modelo de
Gompertz modificado apresentou um bom ajuste aos dados, conforme apresentado na Tabela 2, pois quanto menor o valor do MSE melhor será o ajuste do modelo aos dados experimentais.

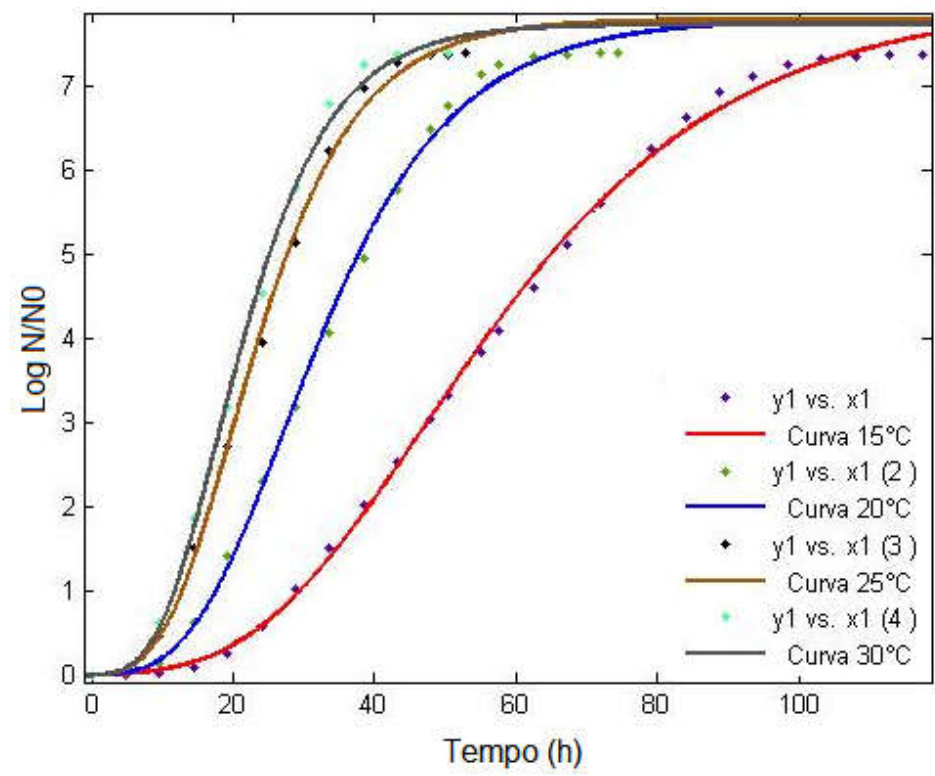

Figura 1. Curvas isotérmicas de crescimento da bactéria Aeromonas hydrophila ajustadas pelo modelo de Gompertz modificado.

Tabela 2. Coefic ientes de ajuste do modelo de Gompertz modific ado aos dados isotérmicos de crescimento da bactéria Aeromonas hydrophila.

\begin{tabular}{ccc}
\hline \multirow{2}{*}{$\mathrm{T}^{\circ} \mathrm{C}$} & \multicolumn{2}{c}{ Coeficientes } \\
\cline { 2 - 3 } & $\mathrm{R}^{2}$ & MSE \\
\hline 15 & 0,9985 & 0,012 \\
20 & 0,9979 & 0,020 \\
25 & 0,9979 & 0,020 \\
30 & 0,9981 & 0,024 \\
\hline
\end{tabular}

O ajuste do modelo de Gompert modificado aos dados de crescimento de 15 a $30^{\circ} \mathrm{C}$ obtiveram coeficientes MSE variando de 0,012 a 0,024 , o que evidencia o bom ajuste deste modelo nas condições estudadas, comprovado pelos valores de $\mathrm{R}^{2}$ que variaram de 0,9979 a 0,9985 .

A Tabela 3 apresenta os parâmetros A, $\lambda$ e $\mu$ que correspondem à duração da fase lag, velocidade específica de crescimento e densidade máxima de população do microrganismo, respectivamente, que foram obtidos dos dados isotérmicos. As figuras 2, 3 e 4 apresentam as curvas de ajuste destes parâmetros em função das temperaturas (modelos secundários). Pode ser observado que as funções lineares que melhor se ajustaram aos parâmetros $\mathrm{A}, \lambda$ e $\mu$ em função das temperaturas isotérmicas foram as Power e Power 1 (Figuras 2, 3 e 4), obtendo bons valores de ajuste conforme os coeficientes MSE e R ${ }^{2}$. 
(LIMA; REIS, ARAGÃO, 2015)

Tabela 3. Parâmetros obtidos das curvas isotérmic as para a cons trução das curvas secundárias de crescimento da bactéria Aeromonas hydrophila.

\begin{tabular}{cc|c|c}
\hline \multirow{2}{*}{ Temperatura } & \multicolumn{3}{|c}{ Parâmetros } \\
\cline { 2 - 4 } & $\mathrm{A}$ & $\lambda$ & $\mu$ \\
\hline $15^{\circ} \mathrm{C}$ & 8,011 & 23,06 & 0,1233 \\
$20^{\circ} \mathrm{C}$ & 7,803 & 13,79 & 0,212 \\
$25^{\circ} \mathrm{C}$ & 7,785 & 10,03 & 0,2958 \\
$30^{\circ} \mathrm{C}$ & 7,715 & 9,25 & 0,3278 \\
\hline
\end{tabular}

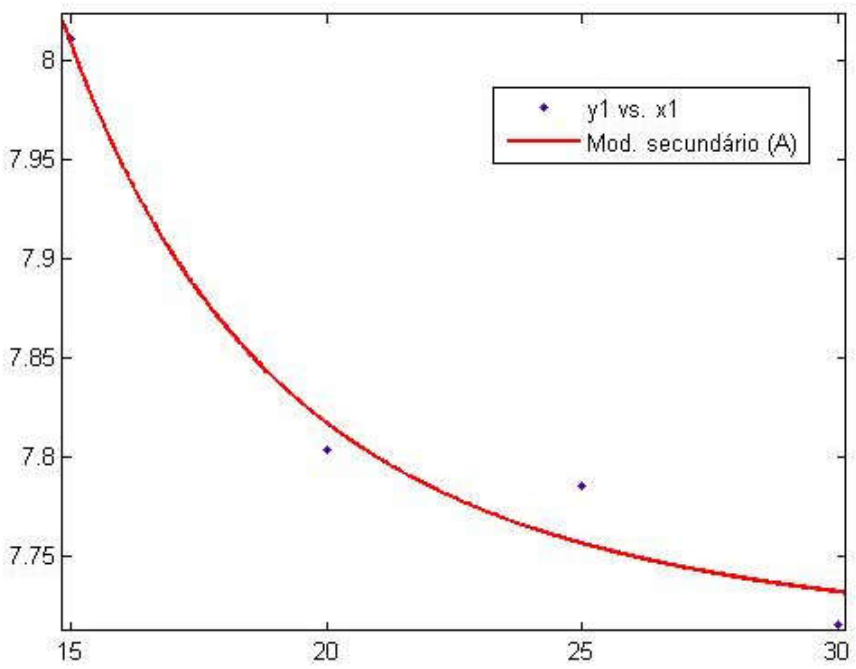

Figura 2. Curva do ajuste de " $A$ " em função das temperaturas. Modelo: (Power) $f(x)=a^{*} x^{\wedge} b+c ; a=3604, b=-3,462$ e $\mathrm{c}=7,704$. Coeficientes de ajuste: $\mathrm{R}^{2}=0,9732 ; \mathrm{MSE}=0,0013$.

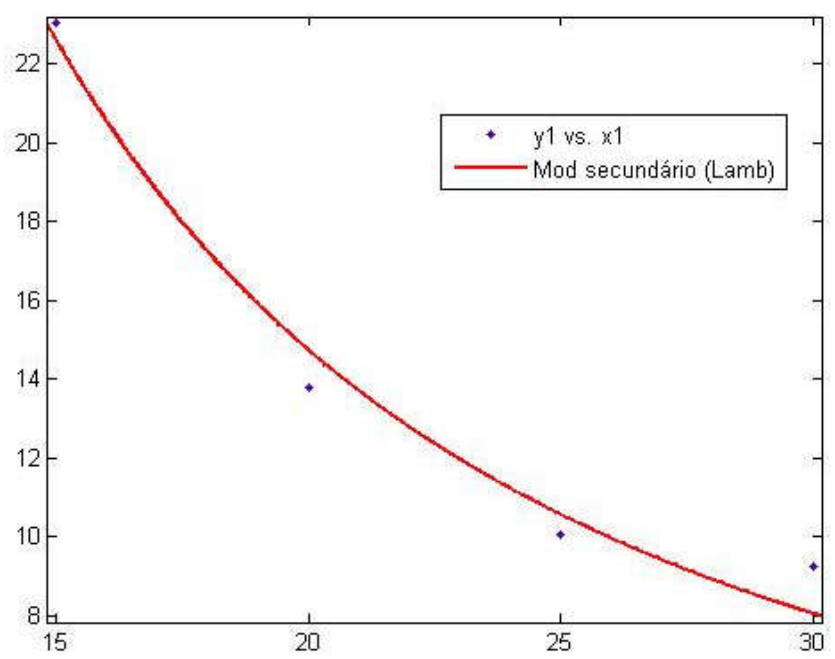

Figura 3. Curva do ajuste de " $\lambda$ ” em função das temperaturas. Modelo: (Power 1) $f(x)=a^{*} x^{\wedge} b ; a=1297$ e $b=-1,495$. Coefic ientes de ajuste: $\mathrm{R}^{2}=0.9767$; MSE: 1,402 . 
(LIMA; REIS, ARAGÃO, 2015)

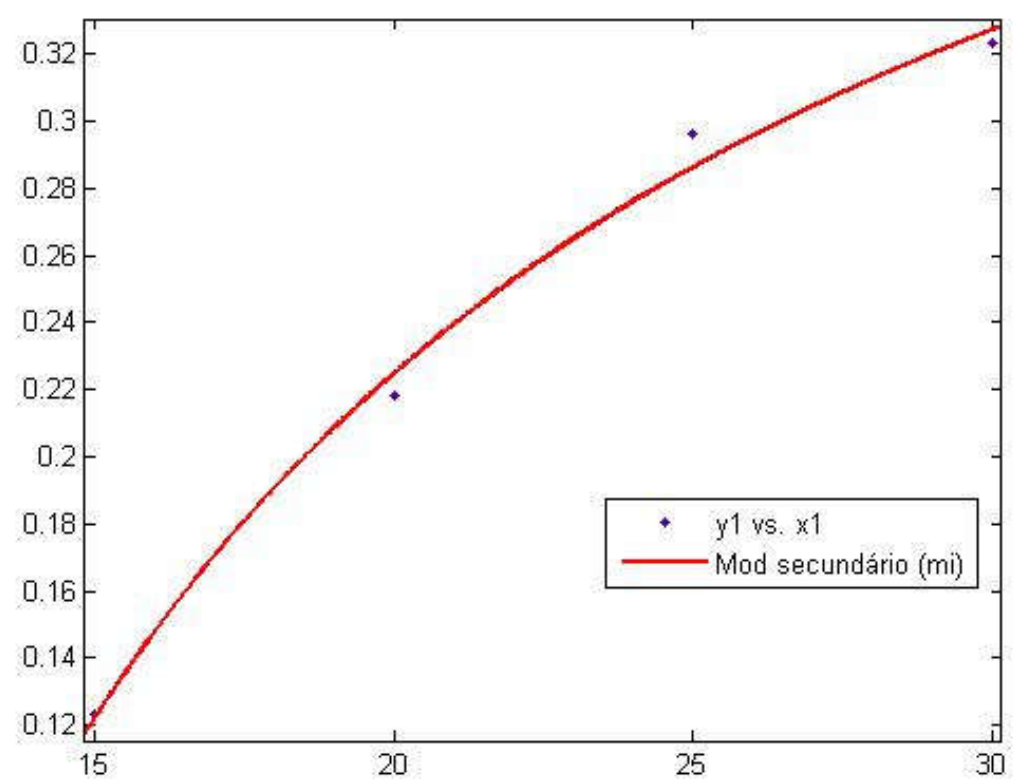

Figura 4. Curva do ajuste de " $\mu$ " em função das temperaturas. Modelo: (Power 1) $f(x)=a^{*} x^{\wedge} b+c ; a=-6,277, b=$ 1,008 e $\mathrm{c}=0,5309$. Coefic ientes de ajuste: $\mathrm{R}^{2}=0.9934 ; \mathrm{MSE}=0,00015$.

Diferentes perfis de temperatura com os respectivos dados de crescimento de $E$. coli divulgados por Fujikawa et al. (2004) foram ajustados com modelos primários e secundários por Corradini e Peleg (2005) utilizando a sua metodologia proposta. Para isso, foram empregados modelos secundários exponenciais no ajuste dos parâmetros obtidos do modelo primário, se concluindo que os modelos exponenciais utilizados apresentaram um bom ajuste podendo ser utilizados para predizer padrõ es de crescimento não isotérmico.

$\mathrm{Na}$ modelagem matemática para a predição do crescimento de Pseudomonas ssp. em condições de temperatura variável realizada por Gaspovic et al. (2008), foram comparados os modelos de Baranyi e Roberts, e Gompertz modificado, como modelos primários de crescimento, e o modelo de Ratkowsky et al. (1983) para ajuste dos parâmetros e obtenção dos modelos secundários, sendo concluído que os dois modelos primários avaliados apresentaram um bom ajuste aos dados de crescimento e produziram resultados semelhantes entre si para a construção do modelo dinâmico.

Neste trabalho se utilizou o modelo de Gompertz modificado para ajuste dos dados de crescimento da bactéria Aeromonas hydrophila (modelo primário) e as funções Power e Power 1 para ajuste dos parâmetros $\mathrm{A}, \lambda$ e $\mu$ (modelos secundários), sendo possível a obtenção de um modelo dinâmico conforme apresentado na Figura 5. Estes resultados confirmam a proposta estabelecida por Corradini \& Peleg (2005) e Corradini et al. (2006), onde é mencionado que modelos lineares podem ser utilizados para o ajuste de parâmetros secundários desde que os dados isotérmicos tenham boa reprodutibilidade. 

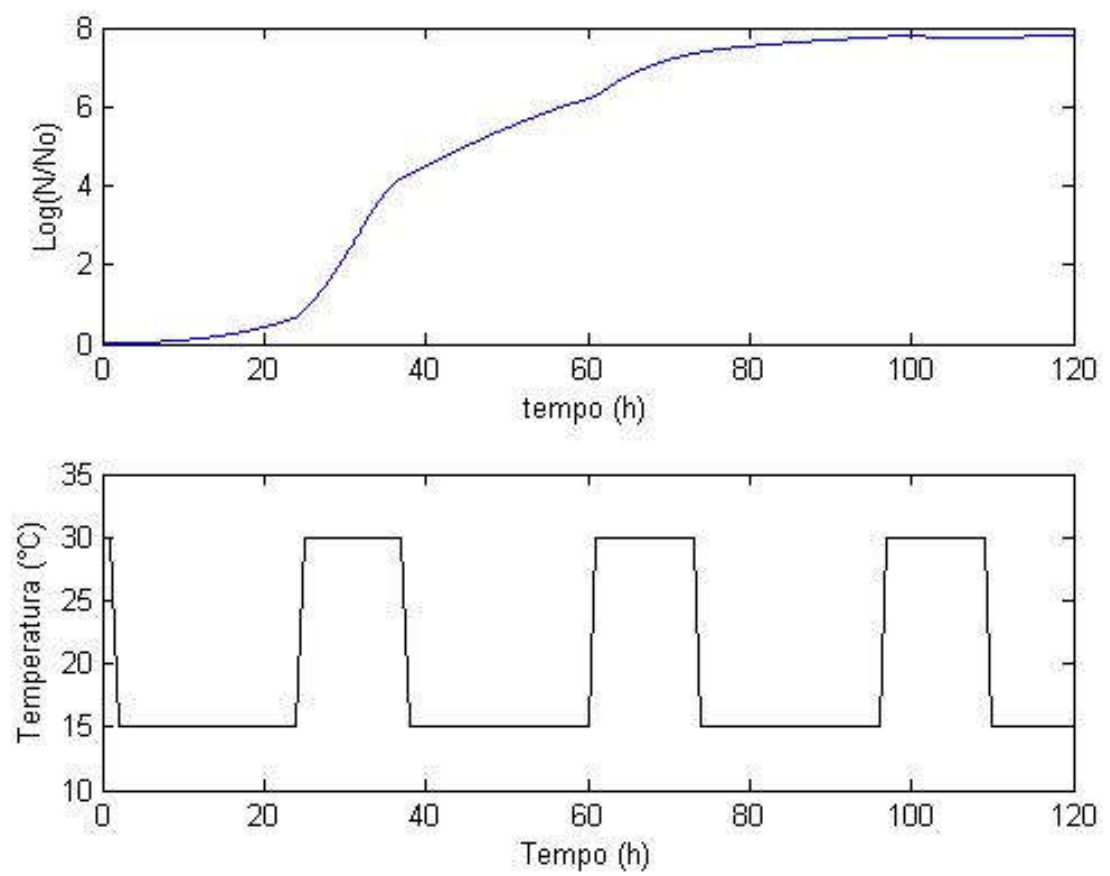

Figura 5. Gráfico do modelo dinâmic o, construído a partir de dados iso térmicos de crescimento da bactéria Aeromonas hydrophila $\mathrm{em} 15,20,2530^{\circ} \mathrm{C}$.

\section{Conclusões}

O modelo de Gompertz modificado se ajustou bem aos dados isotérmicos de crescimento e a utilização das equações "Power" e "Power 1" no ajuste do modelo secundário apresentou bons resultados, sendo possível a obtenção de um modelo dinâmico a partir da metodologia proposta por Corradini e Peleg (2005) e Corradini e colaboradores (2006).

\section{Referências}

Azevedo, V. M.; Morita, M.; Dropa, M.; Cabianca, M. A. A.; Esteves, K. E. E.; Matté, G. R. Matté, M. H. Ocorrência de Aeromonas spp. e Vibrio cholerae em Pesque-Pagues da Região Metropolitana de São Paulo. REVNET DTA. Vol. 3, No. 4, Julho 2003.

Baranyi, J.; Roberts, T. A., Mathematics of Predictive Food Microbiology. International Journal of Food Microbiology, v. 26, p.199218, 1995.
Bobelyn, E.; Hertog, L. A. T. M. M.; Nicolaï, B. M. (2006) Applicability of an enzymatic time temperature integrator as a quality indicator for mushrooms in the distributionchain. Postharvest Biology and Technology 42, 104-114.

Buchanan, R. L. Predictive Microbiology. Trends Food Science Technology, v.4, p.6-11, 1993.

Buchanan, R. L.; Whiting, R. C.; Damert, W. C. When is simple good enough: A comparison of the Gompertz, Baranyi, and three-phase linear models for fitting bacterial growth curves. Food Microbiology, v.14, p.313-326, 1997.

Cayré, M. E.; Vignolo, G.; Garro, O. Modeling lactic acid bacteria growth in vacuum-packaged cooked meat emulsion stored at three temperatures. Food Microbiology, v. 20, p.561-566, 2003.

Cayré, M. E.; Vignolo, G.; Garro, O. Effect of storage temperature and gas permeability of 
packaging film on the growth of lactic acid bacteria and Brochothrix thermosphacta in cooked meat emulsions. Food Mic robiology, v. 22, p.505-512, 2005.

COMBASE Predictor - Base de dados de microrganismos patogênicos. Disponível em: $<$ http://modelling.combase.cc/ComBase Predic tor.aspx $>$ Acessado em 08/09/2011.

Corradini, M. G.; Amezquita, A.; Normand, M. D.; Peleg, M. Modeling and predicting nonisothermal microbial growth using general purpose software. International Journal of Food Microbiology, v.106, p. 223-228, 2006.

Corradini, M. G.; Peleg, M. Estimating nonisothermal bacterial growth in foods from isothermal experiments data. Journal of Applied Microbio logy, v. 99, p 187-200, 2005.

Fujikawa, H.; Kai. A.; Morozumi, S.; A new logistic model for Escherichia coli growth at constant and dynamic temperatures. Food Microbiology, v.21, p.501-509, 2004.

Gaspovic, R.; Kreyenschmidt, J.; Bruckner, S.; Popov, V.; Haque, N. Mathematical modelling for predicting the growth of Pseudomonas spp. in poultry under variable temperature conditions. International Journal of Food Microbiology 127 (2008) 290-297.

Gibson, A. M.; Bratchell, H.; Roberts, T. A. (1987). The effect of sodium chloride and temperature on rate and extent of growth of Clostridium botulinum type $\mathrm{A}$ in pasteurized pork slurry. Applied Bacteriology. 62, 479490.

McMeekin, T. A.; Brown, J. ; Krist, K.; Miles, D.; Neumeyer, K.; Nichols, D. S. ; Olley, J.; Presser, K. ; Ratkowsky, T. D. A.; Ross, M. S.; Soontranon, S. Quantitative Microbiology: A Basis for Food Safety, Emerging Infectious Diseases, v. 3, n 4, 1997.

McMeekin, T. A.; Olley, M. B.; Ross, T., Ratkowsky, D. A., Predictive Microbiology: theory and application. Researches Studies, p. 1-86, 1993.

Nakashima, S. M. K.; André, D. S. ; Franco, B. D. G. M. Revisão: Aspectos Básicos da Microbiologia Preditiva. Brazilian Journal of Food Technology, v. 3, p.41-51, 2000.

Ratkowsky, D. A.; Lowry, R. K.; Mcmeekin, T. A.; Stokes, A. N.; Chandler, R. E. (1983) Model for bacterial culture growth rate through the entire biokinetic temperature range. Journal of Bacteriology 154, 1222-1226.

Ross, T.; McMeekin, T. A. Predictive Microbiology. International Journal of Food Microbiology, v.23, p.41-264, 1994.

Sarmento, C. M. P. Modelagem do crescimento microbiano $e$ avaliação sensorial no estudo da vida de pratele ira da mortadela e da lingüiça defumada em armazenamento is otérmico e não isotérmico. (Tese de Doutorado), Programa de pósgraduação em Engenharia Química. UFSC, Florianópolis, setembro de 2006.

Slongo, A. P.; Rosenthal, A.; Camargo, L. M. Q.; Deliza, R.; Mathias, S. P.; Aragão, G. M. F. Modeling the growth of lactic acid bacteria in sliced ham processed by high hydrostatic pressure. Food Science and Technology, 42 (2009) 303-306.

Van Impe, J. F.; Bart, M. N.; Schellekens, M.; Martens, T.; Baerdemaeker, J. A. Predictive microbiology in a dynamic environment: a system theory approach. International Journal of Food Microbiology, v. 25, p.227249, 1995. 